depolarizations in 18 patients with subarachnoid hemorrhage who underwent surgery to treat an intracranial aneurysm; through the craniotomy, a linear, six-contact electrocorticography recording electrode was placed on the cortex.

Continuous electrocorticographic monitoring was carried out for periods of up to 10 days. Over a combined monitoring time of $2,110 \mathrm{~h}$, a total of 298 spreading depolarizations were detected, in 13 of the 18 patients. A delayed ischemic neurological deficit (DIND) occurred in 7 of these patients an average of 7.8 days after subarachnoid hemorrhage; DINDs were timelocked to new clusters of spreading depolarizations in all cases, and the positive and negative values of such a cluster for predicting DIND were $86 \%$ and $100 \%$, respectively. Delayed cortical infarcts developed in the recording area in four patients; these infarcts followed prolonged electrocorticographic depression associated with spreading depolarizations. Patients who had depression periods $>10 \mathrm{~min}$ had worse outcome on discharge to the rehabilitation unit than those who did not.

The authors conclude that spreading depolarizations are common in cases of major subarachnoid hemorrhage, and that clusters of spreading depolarizations with prolonged depression periods are indicative of progressive brain damage.

Original article Dreier JP et al. (2006) Delayed ischaemic neurological deficits after subarachnoid haemorrhage are associated with clusters of spreading depolarizations. Brain 129: 3224-3237

\section{Arginine growth hormone stimulation test can distinguish between MSA and PD}

Multiple system atrophy (MSA) can be difficult to distinguish from Parkinson's disease (PD). Reduced release of growth hormone $(\mathrm{GH})$ by the pituitary gland in response to arginine has been proposed as a marker of MSA, but current evidence is conflicting. To evaluate the diagnostic accuracy of the arginine $\mathrm{GH}$ stimulation test, Pellecchia et al. measured the response of $\mathrm{GH}$ to arginine in 69 patients with MSA (43 of whom had parkinsonism as the main motor feature and 26 of whom had predominant cerebellar features), 35 patients with PD, and 90 healthy controls. Following the collection of baseline blood samples, $30 \mathrm{~g}$ of intravenous arginine was given to each participant over a period of $30 \mathrm{~min}$; 3 blood samples were subsequently taken at 30 min intervals.

The increase in $\mathrm{GH}$ concentration in response to arginine was significantly lower $(P<0.01)$ in patients with MSA than in either patients with PD or control subjects. Using receiver operating curve analysis, the researchers calculated that the sensitivity and specificity of the arginine $\mathrm{GH}$ stimulation test were highest at a GH concentration of $4 \mu \mathrm{g} / \mathrm{l}$. At this optimum cutoff, the test had a positive predictive value for MSA of $95 \%$, and a sensitivity and specificity of $93 \%$ and $91 \%$, respectively. $\mathrm{GH}$ response to arginine did not correlate with disease duration, MSA motor subtype or response to dopamine. The authors conclude that a reduced $\mathrm{GH}$ response to arginine is an accurate diagnostic marker of MSA.

Original article Pellecchia MT et al. (2006) Multiple system atrophy is distinguished from idiopathic Parkinson's disease by the arginine growth hormone stimulation test. Ann Neurol 60: 611-615

\section{Antiglycolytic compounds: a new class of drugs for treating epilepsy}

Over a third of people affected with epilepsy continue to have seizures despite treatment with antiepileptic drugs or surgery. Dietary manipulation is effective in many of these patients, indicating that the pathways involved in energy metabolism could be targets for future pharmacological intervention. Garriga-Canut and co-workers studied the effect of the glycolytic inhibitor 2-deoxy-D-glucose (2DG) on the progression of epileptogenesis in a rat kindling model of temporal lobe epilepsy, a common drug-resistant form of epilepsy.

Administration of 2DG increased the afterdischarge threshold, and reduced the progression of kindling. This impairment of epileptogenesis downregulated the expression of brain-derived neurotrophic factor (BDNF) and its principal receptor TrkB during seizures, implicating these factors as important in the progression of kindling; the genes encoding BDNF and TrkB have been associated with epileptogenesis in previous studies. The reduced expression of BDNF and TrkB was mediated by the transcription factor neural restrictive silencing factor (NRSF), which alongside the co-repressor $\mathrm{C}$-terminal binding protein (CtBP) acted to 